\title{
Meta-análise do uso de ácido linoleico conjugado na alimentação de suínos
}

\author{
Ines Andretta ${ }^{(1)}$, Paulo Alberto Lovatto(1), Cheila Roberta Lehnen(1), Luciano Hauschild ${ }^{(1)}$ \\ e Carlos Augusto Rigon Rossi(1)
}

\begin{abstract}
(1)Universidade Federal de Santa Maria, Departamento de Zootecnia, CEP 97105-900 Santa Maria, RS. E-mail: ines_andretta@mail.ufsm.br, lovatto@smail.ufsm.br, cheilalehnen@yahoo.com.br, lucianoh@mail.ufsm.br, carlos.rossi@hotmail.com
\end{abstract}

Resumo - O objetivo deste trabalho foi realizar uma meta-análise da associação do ácido linoleico conjugado (CLA) com o desempenho e a qualidade de carcaça e de carne em suínos (Sus scrofa domesticus). A base de dados utilizada contemplou 15 artigos publicados entre 1999 e 2006, e totalizou 216 dietas e 5.223 animais. A meta-análise foi realizada por meio de análises gráficas (para observar coerência biológica dos dados), de correlação (para identificar variáveis correlacionadas) e de variância-covariância. O modelo da análise de variância incluiu apenas as variáveis de carne e carcaça mais correlacionadas com o consumo de CLA pelos animais, além das codificações para os efeitos inter e intra-experimentos. A inclusão do ácido linoleico apresentou correlação negativa com a eficiência alimentar e positiva com o consumo de ração e o ganho de peso dos animais. Não houve alteração do consumo de ração, do ganho de peso e da eficiência alimentar dos suínos. O ácido linoleico conjugado aumentou em $9 \%$ o conteúdo de carne magra na carcaça, e seu consumo variou a espessura média de toucinho. $\mathrm{O}$ ácido linoleico conjugado aumenta o conteúdo de carne magra e reduz a espessura de toucinho na carcaça, sem influenciar o desempenho e a qualidade da carne em suínos.

Termos para indexação: Sus scrofa domesticus, desempenho produtivo, metabolismo, nutrição animal, qualidade de carcaça.

\section{Meta-analysis of conjugated linoleic acid use in pigs feeding}

\begin{abstract}
This work aimed at meta-analyzing the association of conjugated linoleic acid (CLA) with the performance and the carcass and meat quality in pigs (Sus scrofa domesticus). The database used assembled 15 scientific papers, published from 1999 to 2006, comprising 216 diets and 5,223 animals. Meta-analysis was carried out by graphical examination (to observe biological coherency), correlation (to identify correlated variables) and variance-covariance analysis of the data. The analysis of variance included only meat and carcass variables with stronger correlation to CLA consumption by the animals, and encodings to inter and intra-experimental effects. The inclusion of CLA in diets showed a negative correlation with feed efficiency and a positive correlation with feed intake and weight gain. Conjugated linoleic acid-suplemented diets for pigs did not affect feed intake, weight gain and feed efficiency. Conjugated linoleic acid increased the lean meat content in the carcass in $9 \%$. Average backfat thickness also varied according to conjugated linoleic acid consumption. Conjugated linoleic acid increases lean meat content and reduces backfat thickness, without influencing performance and meat quality in pigs.
\end{abstract}

Index terms: Sus scrofa domesticus, productive performance, metabolism, animal nutrition, carcass quality.

\section{Introdução}

Os ácidos graxos são nutrientes fundamentais na alimentação animal pelo potencial energético e participação na estrutura celular. A essencialidade de certos ácidos graxos é determinada pela incapacidade de alguns animais de sintetizá-los a partir de precursores estruturalmente mais simples. Entre os ácidos graxos essenciais está o ácido linoleico.

O termo ácido linoleico conjugado (CLA) refere-se a uma mistura de isômeros geométricos e posicionais desse ácido, com duplas ligações conjugadas. O CLA é produzido naturalmente por bactérias fermentativas ruminais, pela bio-hidrogenação incompleta de ácidos graxos poli-insaturados provenientes da dieta (Santos-Zago et al., 2008). Após a absorção, o CLA é incorporado aos tecidos e é encontrado na carne de ruminantes e em produtos lácteos derivados (Lin et al., 1995). Recentemente, o CLA tem sido objeto de muitas pesquisas, principalmente por sua relação com a saúde humana. Entre os benefícios atribuídos ao CLA, destacam-se a anticarcinogênese, antiarterosclerose,

Pesq. agropec. bras., Brasília, v.44, n.7, p.754-760, jul. 2009 
inibição de radicais livres, alteração na composição e no metabolismo do tecido adiposo, melhoria no perfil lipídico sanguíneo, imunomodulação e atividades antibacteriana e antidiabética (Funck et al., 2006; Santos-Zago et al., 2008).

Os suínos possuem microbiota gástrica reduzida e pouco funcional. A alta taxa de passagem do conteúdo estomacal aliada ao baixo potencial gástrico de hidrogenação de ácidos graxos limita a produção de CLA nessa espécie (Dugan et al., 2004). No entanto, a carne suína poderia, fisiologicamente, ser uma fonte expressiva de CLA para a alimentação humana, desde que os animais recebessem o suplemento por via alimentar. Em condições adequadas, a suplementação com CLA, em dietas para animais, poderia aumentar os teores desse ácido graxo em alimentos destinados ao consumo humano (Wiegand et al., 2002) e, assim, servir como coadjuvante na prevenção e no controle de doenças crônicas e associadas.

Muitos trabalhos vêm sendo conduzidos com o intuito de avaliar os efeitos do CLA na composição corporal de animais de produção como aves (Zanini et al., 2008) e peixes (Santos et al., 2007). A associação do CLA com alterações metabólicas também tem sido objeto de diversas pesquisas. Porém, os efeitos do CLA, especialmente em suínos, ainda não estão bem esclarecidos. Alguns estudos têm indicações de que o CLA pode ser utilizado como suplemento nutricional para suínos (Dugan et al., 2004). Porém, os resultados dessas pesquisas ainda são inconstantes e pouco conclusivos. O CLA foi associado, por diversos autores, ao aumento na eficiência alimentar dos suínos (Ostrowska et al., 1999; Thiel-Cooper et al., 2001; Wiegand et al., 2001; Weber et al., 2006). Porém, essas alterações na eficiência alimentar não foram observadas em outros trabalhos (O'Quinn et al., 2000; Eggert et al., 2001; Ramsay et al., 2001; Weber et al., 2001, 2006). Os efeitos do CLA, sobre o ganho de peso e sobre algumas características de carne e carcaça, também variam nos estudos prévios com suínos (Dugan et al., 2004). Uma meta-análise de dados das publicações disponíveis poderia integrar as diferentes variáveis experimentais e permitir estabelecer respostas sistêmicas e ajustadas à diversidade experimental.

Este trabalho teve o objetivo de determinar, por meio de meta-análise, a relação entre o fornecimento de CLA na dieta e o desempenho e características de carcaça e de carne, em suínos.

\section{Material e Métodos}

O trabalho foi realizado no Setor de Suínos do Departamento de Zootecnia da Universidade Federal de Santa Maria. A base de dados utilizada para realização da meta-análise foi composta por 15 artigos (Ostrowska et al., 1999; O'Quinn et al., 2000; Bassaganya-Riera et al., 2001; Eggert et al., 2001; Ramsay et al., 2001; Thiel-Cooper et al., 2001; Weber et al., 2001; Wiegand et al., 2001; D'Souza \& Mullan, 2002; Gatlin et al., 2002; Joo et al., 2002; Wiegand et al., 2002; Corino et al., 2003; Ostrowska et al., 2003; Weber et al., 2006). Esses artigos foram publicados em periódicos internacionais (British Journal of Nutrition, Journal of Nutrition, Meat Science e Journal of Animal Science) entre 1999 e 2006. Das publicações utilizadas nessa base, dez tiveram origem em institutos americanos. As demais tomaram por base estudos realizados na Austrália (3), Itália (1) e Coreia (1). Por ocasião da seleção dos artigos a serem inseridos na base de dados, não foram encontrados trabalhos nacionais indexados, o que justifica a ausência dessas referências nesta meta-análise.

Os principais critérios de seleção das publicações foram: adição de CLA nas dietas, genética (raças ou cruzamentos "industriais"), fase alimentar (creche, crescimento ou terminação), avaliações de desempenho e qualidade de carcaça e carne. A metodologia para a definição das variáveis dependentes e independentes e para a codagem dos dados seguiu as proposições descritas na literatura (Lovatto et al., 2007; Sauvant et al., 2008).

As informações relativas às variáveis a serem analisadas foram selecionadas nas seções de material e métodos e de resultados, de cada artigo, e inseridas em uma base de dados elaborada em planilha Excel (Microsoft). As variáveis analisadas foram relativas a características experimentais (período experimental e de fornecimento do CLA, inclusão do CLA nas dietas, idade, peso, sexo e lotação), à composição nutricional das dietas, ao desempenho (consumo de ração, ganho de peso e eficiência alimentar) e a características de carcaça (rendimento, peso, comprimento, área de lombo, carne magra, perda de água e espessura de toucinho) e de carne (marmoreio, consistência, lipídeos, $\mathrm{pH}$ e tonalidade).

A base foi composta de 216 dietas e 5.223 animais, com média de 24 animais por tratamento. O peso dos animais no início do experimento foi, em 
média, de 42,4 quilogramas. O peso inicial foi menor que $10 \mathrm{~kg}$ em apenas três trabalhos, e dois deles foram prolongados até a fase de terminação. Em cinco trabalhos estudou-se a suplementação das dietas com CLA, durante o crescimento e terminação, e em sete trabalhos, apenas na fase de terminação dos animais.

Os principais ingredientes utilizados nas dietas e suas estatísticas descritivas estão apresentados na Tabela 1. O milho e o farelo de soja foram as principais fontes energética e proteica, utilizados em 70 e $93 \%$ das dietas, respectivamente. $\mathrm{O}$ óleo de soja participou da formulação de $48 \%$ das dietas. A concentração média de CLA nos aditivos utilizados como fonte do composto nas dietas experimentais foi de 58,8\% (de 55 a $65 \%$ ). O nível de inclusão médio desses produtos foi de $1,49 \%$, com variação de 0 a $10 \%$ das dietas. O período médio de fornecimento das rações experimentais foi de 40 dias (variação de 7 a 95). A média do número de animais por baia foi 5 (variou de 1 a 25), com espaçamento médio de $1,28 \mathrm{~m}^{2}$ por animal.

A meta-análise foi realizada segundo Lovatto et al. (2007) e Sauvant et al. (2008) e seguiu três análises sequenciais: gráfica, para observar a coerência biológica dos dados; de correlação entre as diversas variáveis, para identificar os fatores correlacionados na base de dados; e de variância. Foram utilizados, nos modelos para as análises de variância e covariância, as variáveis de carne e carcaça mais correlacionadas com o consumo de CLA pelos animais e as codificações para os efeitos inter e intraexperimentos (Lovatto et al., 2007). As equações de regressão foram obtidas por meio da análise de variância-covariância com uso do GLM (modelo linear generalizado). Todas as análises foram realizadas com o programa MINITAB (Minitab, 2007).

\section{Resultados e Discussão}

A Tabela 2 apresenta o desempenho dos suínos, de acordo com a suplementação das dietas com CLA. De modo geral, embora com valores baixos, o conteúdo de CLA nas dietas apresentou correlação positiva com o consumo de ração $(\mathrm{CR})(0,26)$ e com o ganho de peso (GP) $(0,14)$. Porém, na eficiência alimentar (EF), o comportamento foi inverso, com correlação de $-0,24$ para a EF observada e de $-0,26$ para a EF calculada. A correlação positiva do $\mathrm{CR}$ com o teor de CLA nas dietas pode ser explicada pela relação entre esse componente e os teores plasmáticos de leptina (Santos-Zago et al., 2008). Essa proteína circulante é produzida proporcionalmente pela massa de tecido adiposo e exerce um papel importante no controle da ingestão de alimentos (Pelleymounter et al., 1995). Dessa forma, o efeito redutor de gordura corporal do CLA (Santos-Zago et al., 2008) resultaria em menores concentrações plasmáticas de leptina. O incremento no CR estaria, portanto, associado a teores plasmáticos mais baixos de leptina, o que reduziria a sensação de saciedade nos animais.

Tabela 1. Principais ingredientes e composição média das dietas.

\begin{tabular}{|c|c|c|c|c|c|}
\hline Ingrediente & № de dietas & Média & Mínimo & Máximo & $\mathrm{CV}(\%)$ \\
\hline & \multicolumn{5}{|c|}{ Percentagem na matéria natural } \\
\hline Milho & 152 & 63,44 & 31,57 & 85,01 & 21,8 \\
\hline Farelo de soja & 200 & 18,91 & 8,25 & 31,16 & 33,5 \\
\hline Trigo & 51 & 65,80 & 13,00 & 69,10 & 1,10 \\
\hline Leite ou soro de leite em pó & 64 & 15,37 & 10,00 & 22,00 & 20,2 \\
\hline Plasma desidratado & 24 & 5,75 & 4,00 & 7,50 & 25,3 \\
\hline Fosfato bicálcico & 33 & 1,73 & 0,51 & 2,60 & 38,7 \\
\hline Fosfato monocálcico & 167 & 2,03 & 0,76 & 2,50 & 37,9 \\
\hline Óleo vegetal & 104 & 1,93 & 0,50 & 3,33 & 52,6 \\
\hline Calcário & 200 & 1,00 & 0,12 & 2,50 & 58,6 \\
\hline Sal & 121 & 0,34 & 0,15 & 0,50 & 31,3 \\
\hline \multirow[t]{2}{*}{ Suplemento vitamínico mineral } & 195 & 0,29 & 0,15 & 0,51 & 30,7 \\
\hline & \multicolumn{5}{|c|}{ Composição das dietas } \\
\hline Energia digestível $\left(\mathrm{kcal} \mathrm{kg}^{-1}\right)$ & 87 & 3.411 & 2.624 & 3.500 & 4,53 \\
\hline Energia metabolizável $\left(\mathrm{kcal} \mathrm{kg}^{-1}\right)$ & 79 & 3.552 & 3.340 & 3.804 & 5,09 \\
\hline Proteína bruta $(\%)$ & 180 & 18,84 & 15,10 & 24,18 & 10,3 \\
\hline Cálcio $(\%)$ & 114 & 0,78 & 0,50 & 1,05 & 19,0 \\
\hline Fósforo total (\%) & 114 & 0,67 & 0,42 & 0,87 & 19,9 \\
\hline Lisina $(\%)$ & 152 & 1,16 & 0,62 & 1,90 & 28,6 \\
\hline Metionina + cistina $(\%)$ & 32 & 0,84 & 0,71 & 0,96 & 10,7 \\
\hline
\end{tabular}

Pesq. agropec. bras., Brasília, v.44, n.7, p.754-760, jul. 2009 
Segundo a análise de variância, o desempenho (CR, EF e GP) não diferiu ( $\mathrm{p}>0,05)$ entre os animais que receberam dietas com CLA e os animais não

Tabela 2. Desempenho dos suínos, em função da suplementação das dietas com ácido linoleico conjugado (CLA) e correlação das variáveis com a inclusão de níveis de CLA nas rações.

\begin{tabular}{|c|c|c|c|}
\hline Tipo de dieta & Média & $\mathrm{DPR}^{(1)}$ & Correlação \\
\hline & \multicolumn{3}{|c|}{ Consumo diário de racão $(\mathrm{kg})$} \\
\hline Controle & $2,030^{\mathrm{ns}}$ & 0,362 & $0,258^{* *}$ \\
\hline \multirow[t]{2}{*}{ Dietas com CLA } & $2,021^{\mathrm{ns}}$ & - & - \\
\hline & \multicolumn{3}{|c|}{ Ganho diário de peso $(\mathrm{kg})$} \\
\hline Controle & $0,785^{\mathrm{ns}}$ & 0,148 & $0,140^{*}$ \\
\hline \multirow[t]{2}{*}{ Dietas com CLA } & $0,795^{\text {ns }}$ & - & - \\
\hline & \multicolumn{3}{|c|}{ Eficiência alimentar (observada) } \\
\hline Controle & $0,454^{\mathrm{ns}}$ & 0,080 & $-0,238^{* *}$ \\
\hline \multirow[t]{2}{*}{ Dietas com CLA } & $0,465^{\mathrm{ns}}$ & - & - \\
\hline & \multicolumn{3}{|c|}{ Eficiência alimentar (calculada) } \\
\hline Controle & $0,455^{\mathrm{ns}}$ & 0,078 & $-0,257^{* *}$ \\
\hline Dietas com CLA & $0,466^{\mathrm{ns}}$ & - & - \\
\hline
\end{tabular}

suplementados. Alguns autores da base de dados relataram que a suplementação com CLA não exerce influência sobre o GP de suínos (Ostrowska et al., 1999; Ramsay et al., 2001; Weber et al., 2001; Wiegand et al., 2002; Ostrowska et al., 2003). Contudo, esses resultados diferem dos observados em outras pesquisas. Ao trabalhar com suplementação de dietas com uma mistura de ácidos graxos com CLA, Thiel-Cooper et al. (2001) verificaram um aumento linear no GP, em função da dose utilizada. Um comportamento quadrático foi observado no GP por Bassaganya-Riera et al. (2001).

Assim como no GP, o efeito do CLA sobre a EF não está evidente nos trabalhos utilizados na base de dados. Alguns trabalhos utilizados nesta meta-análise não observaram alteração na EF dos animais (O'Quinn et al., 2000; Eggert et al., 2001; Ramsay et al., 2001; Weber et al., 2001; Weber et al., 2006). Contudo, diversos autores associaram o CLA a um aumento na EF de suínos (Ostrowska et al., 1999; Thiel-Cooper

Tabela 3. Equações obtidas por análise de covariância contínua, para as respostas de desempenho e características de carcaça e carne, em suínos ${ }^{(1)}$.

\begin{tabular}{|c|c|c|c|c|}
\hline $\begin{array}{l}\text { Resposta de desempenho e características } \\
\text { de carcaça e carne }\end{array}$ & Covariáveis & DPR & Equação & $\mathrm{R}^{2}$ \\
\hline & \multicolumn{4}{|c|}{ Desempenho } \\
\hline \multirow{4}{*}{ Consumo diário de ração $(\mathrm{kg})$} & CLA & 0,36 & 2,34-0,0304 CLA & 87,49 \\
\hline & CLA e PVm & 0,26 & $1,19-0,021 \mathrm{CLA}+0,020 \mathrm{PVm}$ & 93,31 \\
\hline & CLA, PB & 0,21 & 5,57 - 0,023 CLA - 0,174 PB & 95,74 \\
\hline & CLA, LIS & 0,29 & $3,90+0,079$ CLA -1,37 LIS & 91,52 \\
\hline \multirow{4}{*}{ Ganho diário de peso $(\mathrm{kg})$} & CLA & 0,18 & 0,884 - 0,017 CLA & 46,72 \\
\hline & CLA e PVm & 0,14 & $0,500-0,006$ CLA $+0,006$ PVm & 67,66 \\
\hline & CLA, PB & 0,14 & 2,35 - 0,008 CLA - 0,081 PB & 68,35 \\
\hline & CLA, LIS & 0,15 & $1,478+0,006$ CLA - 0,529 LIS & 68,74 \\
\hline \multirow{4}{*}{ Eficiência alimentar } & CLA & 0,08 & $0,418+0,003$ CLA & 74,75 \\
\hline & CLA e PVm & 0,06 & $0,625+0,001 \mathrm{CLA}-0,003$ PVm & 85,26 \\
\hline & CLA, PB & 0,06 & $-0,355+0,0004 \mathrm{CLA}+0,042 \mathrm{~PB}$ & 88,64 \\
\hline & CLA, LIS & 0,05 & $0,0205+0,008$ CLA + 0,364 LIS & 91,51 \\
\hline \multirow{5}{*}{ ET na décima costela ( $\mathrm{mm})$} & \multicolumn{4}{|c|}{ Avaliações de carcaça e carne } \\
\hline & CLA & 6,85 & $18,79+1,148$ CLA & 44,32 \\
\hline & CLA e PVm & 6,99 & $-2,62+0,577$ CLA + 0,226 PVm & 63,34 \\
\hline & CLA e DIAS & 6,91 & $19,40+1,109$ CLA - 0,020 DIAS & 44,57 \\
\hline & CLA (mg) & 7,35 & 20,18 - 0,060 CLA (mg) & 50,55 \\
\hline ET na última costela (mm) & CLA (mg) & 8,76 & 22,09 - 0,037 CLA (mg) & 62,71 \\
\hline ET média calculada (mm) & CLA (mg) & 8,93 & 24,22 - 0,069 CLA (mg) & 52,66 \\
\hline Área de lombo $\left(\mathrm{cm}^{2}\right)$ & CLA (mg) & 3,44 & $35,58+0,004$ CLA (mg) & 88,73 \\
\hline Carne magra $(\%)$ & CLA (mg) & 4,73 & $57,25+0,003$ CLA (mg) & 67,32 \\
\hline Escore de cor visual & CLA (mg) & 0,11 & 2,62 - 0,009 CLA (mg) & 66,28 \\
\hline Marmoreio & CLA (mg) & 0,32 & $2,18-0,019$ CLA (mg) & 75,43 \\
\hline Escore de consistência & CLA (mg) & 0,28 & 2,88 - 0,026 CLA (mg) & 61,54 \\
\hline
\end{tabular}


et al., 2001; Wiegand et al., 2001; Weber et al., 2006). A equação obtida por análise de covariância contínua (Tabela 3) mostra incremento na EF, à medida que se aumentou a inclusão de CLA nas dietas. Alterações na partição dos nutrientes e decréscimo na síntese lipídica são os principais efeitos biológicos do CLA, possivelmente relacionados à EF (Santos-Zago et al., 2008). Os efeitos do CLA na EF e nas demais variáveis de desempenho dependem da quantidade e da composição de isômeros utilizados na mistura em teste (Wang \& Jones, 2004).

De modo geral, as equações que melhor explicaram o ganho de peso dos animais foram as que apresentaram, como covariáveis, o percentual de proteína bruta ou de lisina na dieta, além do teor de CLA (Tabela 3). Estudos anteriores indicaram que o tipo de proteína da dieta pode influenciar a atividade do CLA em ratos (Akahoshi et al., 2004). Os resultados da presente meta-análise evidenciam que a combinação apropriada entre o CLA e os diferentes componentes nutricionais deve ser considerada, também, para suínos e merece atenção em pesquisas futuras.

A correlação entre carne magra na carcaça e teor de CLA nas dietas foi de 0,59 (Tabela 4). Da mesma forma, um incremento no percentual de carne magra pode ser esperado pelo aumento no período de fornecimento do CLA. O consumo de CLA apresentou correlação negativa com as espessuras de toucinho na altura da primeira, décima e última costela. A correlação entre espessura de toucinho média e consumo de CLA apresentou comportamento semelhante ao das variáveis reportadas anteriormente.

A suplementação de dietas com CLA não alterou a área de olho de lombo e a perda de água após 24 horas (Tabela 5). O fornecimento de CLA também não interferiu no peso, comprimento e rendimento de carcaça nos suínos. As características de carne avaliadas (cor, marmoreio, consistência, tonalidade, percentual de lipídeos e $\mathrm{pH}$ ) também não foram influenciadas pela adição de CLA na dieta. Porém, foi observada redução de $6 \%$ na espessura de toucinho, na altura da décima costela, e de $7 \%$ na altura da última costela. O CLA também aumentou em cerca de $9 \%$ o conteúdo de carne magra na carcaça dos suínos.

A equação obtida por análise de covariância contínua (Tabela 3) também mostra que a espessura de toucinho média varia de acordo com o consumo de CLA. Para cada miligrama de CLA consumido durante as fases de crescimento e terminação, houve redução de $0,06 \mathrm{~mm}$ na espessura de toucinho, na altura da décima

Tabela 4. Coeficientes de correlação entre as variáveis de carcaça e carne, em suínos, e o teor de ácido linoleico conjugado (CLA) nas dietas, o período de fornecimento do o composto e o consumo de CLA pelos animais.

\begin{tabular}{|c|c|c|c|}
\hline Características de carcaça e carne & Teor de CLA nas dietas (\%) & Período (dias) & Consumo de CLA (mg) \\
\hline & \multicolumn{3}{|c|}{ Características de carcaca } \\
\hline Peso quente $(\mathrm{kg})$ & $0,054^{\mathrm{ns}}$ & $0,069^{\text {ns }}$ & $0,112^{\mathrm{ns}}$ \\
\hline Rendimento de carcaça (\%) & $0,358^{*}$ & $0,084^{\mathrm{ns}}$ & $0,420^{* *}$ \\
\hline ET na décima costela (mm) & $0,054^{\mathrm{ns}}$ & $-0,158^{\mathrm{ns}}$ & $-0,287^{\mathrm{ns}}$ \\
\hline ET na primeira costela $(\mathrm{mm})$ & $0,043^{\text {ns }}$ & $-0,067^{\mathrm{ns}}$ & $-0,285^{\mathrm{ns}}$ \\
\hline ET na última costela $(\mathrm{mm})$ & $0,048^{\text {ns }}$ & $-0,232^{\mathrm{ns}}$ & $-0,314^{\mathrm{ns}}$ \\
\hline ET média calculada (mm) & $0,005^{\mathrm{ns}}$ & $-0,088^{\mathrm{ns}}$ & $-0,244^{\mathrm{ns}}$ \\
\hline Área de lombo $\left(\mathrm{cm}^{2}\right)$ & $-0,217^{\mathrm{ns}}$ & $0,067^{\mathrm{ns}}$ & $-0,180^{\mathrm{ns}}$ \\
\hline Comprimento de carcaça $(\mathrm{cm})$ & $-0,288^{\mathrm{ns}}$ & $-0,382^{\mathrm{ns}}$ & $-0,367^{\mathrm{ns}}$ \\
\hline Carne magra $(\%)$ & $0,586^{* * *}$ & $0,237^{\mathrm{ns}}$ & $0,612^{* * *}$ \\
\hline Perda de água em 24 horas (\%) & $0,038^{\mathrm{ns}}$ & $0,062^{\text {ns }}$ & $0,073^{\mathrm{ns}}$ \\
\hline \multicolumn{4}{|c|}{ Características de carne } \\
\hline Cor & $0,025^{\mathrm{ns}}$ & $-0,080^{\mathrm{ns}}$ & $-0,292^{\mathrm{ns}}$ \\
\hline Marmoreio & $-0,032^{\mathrm{ns}}$ & $0,269^{\text {ns }}$ & $0,230^{\mathrm{ns}}$ \\
\hline Lipídeos (\%) & $0,131^{\mathrm{ns}}$ & $-0,087^{\mathrm{ns}}$ & $0,109^{\mathrm{ns}}$ \\
\hline Consistência & $0,066^{\mathrm{ns}}$ & $0,003^{\mathrm{ns}}$ & $-0,204^{\mathrm{ns}}$ \\
\hline Tonalidades claras $\left(\mathrm{L}^{*}\right)$ & $0,141^{\mathrm{ns}}$ & $-0,013^{\mathrm{ns}}$ & $-0,054^{\mathrm{ns}}$ \\
\hline Tonalidades vermelhas $\left(\mathrm{a}^{*}\right)$ & $-0,130^{\mathrm{ns}}$ & $-0,079^{\mathrm{ns}}$ & $-0,320^{\mathrm{ns}}$ \\
\hline Tonalidades amareladas $\left(\mathrm{b}^{*}\right)$ & $-0,312^{\mathrm{ns}}$ & $0,258^{\mathrm{ns}}$ & $-0,067^{\mathrm{ns}}$ \\
\hline Tonalidade $a^{*} b^{*-1}$ & $0,164^{\mathrm{ns}}$ & $-0,205^{\mathrm{ns}}$ & $-0,140^{\mathrm{ns}}$ \\
\hline $\mathrm{pH}(45 \mathrm{~min})$ & $-0,028^{\mathrm{ns}}$ & $-0,304^{\mathrm{ns}}$ & $-0,380^{\mathrm{ns}}$ \\
\hline pH (24 horas) & $0,300^{\mathrm{ns}}$ & $0,217^{\mathrm{ns}}$ & $0,141^{\mathrm{ns}}$ \\
\hline
\end{tabular}


costela (ET10 $=20,18-0,06 \mathrm{mg}$ de CLA) e de cerca de $0,04 \mathrm{~mm}$ na medida avaliada na altura da última costela (ETult $=22,09-0,037 \mathrm{mg}$ de CLA).

Alterações na composição corporal de animais alimentados com dietas com CLA foram descritas em suínos (Ostrowska et al., 1999; Ramsay et al., 2001; Thiel-Cooper et al., 2001; Wiegand et al., 2001; Wiegand et al., 2002; Corino et al., 2003; Ostrowska et al., 2003; Weber et al., 2006) e, também, em outras espécies como aves (Zanini et al., 2008) e peixes (Santos et al., 2007). Porém, os mecanismos bioquímicos de ação dos diferentes isômeros do CLA ainda não estão adequadamente elucidados e comprovados, e a maioria desses dados tem origem em trabalhos experimentais com camundongos ou in vitro.

O CLA modula processos bioquímicos e fisiológicos envolvidos na mobilização e armazenagem dos lipídeos (Santos-Zago et al., 2008). Algumas das hipóteses metabólicas, para explicar os efeitos do CLA sobre a composição corporal dos animais, incluem um controle da expressão de genes envolvidos na diferenciação dos pré-adipócitos em células maduras

Tabela 5. Médias das avaliações das características de carcaça e carne de suínos, alimentados com dieta suplementada ou não (controle) com ácido linoleico conjugado (CLA).

\begin{tabular}{|c|c|c|c|}
\hline Característica de carcaça e carne $^{(1)}$ & Controle & CLA & $\mathrm{DPR}^{(2)}$ \\
\hline & \multicolumn{3}{|c|}{ Características de carcaça } \\
\hline Peso quente (kg) & 76,6 & $75,0^{\mathrm{ns}}$ & 0,9 \\
\hline Rendimento de carcaça (\%) & 74,7 & $75,1^{\mathrm{ns}}$ & 0,1 \\
\hline ET na altura da décima costela $(\mathrm{mm})$ & 12,6 & $11,8^{* * *}$ & 0,8 \\
\hline ET na altura da primeira costela $(\mathrm{mm})$ & 19,7 & $21,3^{\mathrm{ns}}$ & 1,4 \\
\hline ET na altura da última costela $(\mathrm{mm})$ & 16,8 & $15,6^{* *}$ & 0,5 \\
\hline ET média calculada (mm) & 14,0 & $13,6^{\mathrm{ns}}$ & 0,6 \\
\hline Área de lombo $\left(\mathrm{cm}^{2}\right)$ & 38,2 & $35,9^{\text {ns }}$ & 2,3 \\
\hline Comprimento de carc & 75,7 & $69,3^{\text {ns }}$ & 0,7 \\
\hline Carne magra $(\%)$ & 55,3 & $60,4^{*}$ & 0,4 \\
\hline \multirow[t]{2}{*}{ Perda de água em 24 horas (\%) } & 4,5 & $4,4^{\mathrm{ns}}$ & 1,1 \\
\hline & \multicolumn{3}{|c|}{ Características de carne } \\
\hline Cor & 2,46 & $2,46^{\mathrm{ns}}$ & 0,11 \\
\hline Marmor & 1,71 & $1,98^{\mathrm{ns}}$ & 0,30 \\
\hline Lipíd & 8,05 & $9,76^{\mathrm{ns}}$ & 1,71 \\
\hline ência & 2,42 & $2,51^{\mathrm{ns}}$ & 0,58 \\
\hline Tonalidades clar & 48,50 & $47,50^{\mathrm{ns}}$ & 2,90 \\
\hline Tonalidades vermelhas $\left(\mathrm{a}^{*}\right)$ & 7,24 & $7,13^{\mathrm{ns}}$ & 2,27 \\
\hline Tonalidades amareladas (b*) & 8,47 & $7,72^{\text {ns }}$ & 1,02 \\
\hline Tonalidades $\mathrm{a}^{*} \mathrm{~b}^{*-1}$ & 1,06 & $1,17^{\mathrm{ns}}$ & 0,41 \\
\hline $\mathrm{pH}(45 \mathrm{~min})$ & 6,26 & $6,29^{\mathrm{ns}}$ & 0,14 \\
\hline $\mathrm{pH}$ (24 horas) & 5,52 & $5,56^{\mathrm{ns}}$ & 0,08 \\
\hline
\end{tabular}

${ }^{(1)} \mathrm{ET}$, espessura de toucinho. ${ }^{(2)}$ Desvio-padrão residual. ns Não significativo. $*, * * \mathrm{e}^{* * *}$ Significativo a 5,1 e $0,1 \%$ de probabilidade, respectivamente, pelo teste $\mathrm{F}$.
(Santos-Zago et al., 2008). Assim, esses mecanismos estariam associados, concomitantemente, à redução na lipogênese e à potencialização da oxidação de ácidos graxos, tanto nos músculos quanto no tecido adiposo (Wang \& Jones, 2004; Santos-Zago et al., 2008). Como a deposição de reservas se dá a partir do balanço positivo entre síntese e degradação lipídica, os efeitos do CLA na composição da carcaça estão relacionados ao acréscimo na deposição de tecido magro em detrimento da gordura. Em suínos, essa relação pode ser alterada em cerca de $31 \%$ pelo CLA (Ostrowska et al., 1999).

A meta-análise realizada neste trabalho permitiu abordar, de forma sistemática, a associação do CLA com as alterações metabólicas em suínos. Os resultados desta meta-análise apontam para a possibilidade de se explorar a relação do CLA com os diversos componentes nutricionais das dietas.

\section{Conclusões}

1. O uso de ácido linoleico conjugado como aditivo nutricional nas dietas para suínos em crescimento não altera o desempenho dos animais.

2. A adição de ácido linoleico conjugado aumenta o conteúdo de carne magra na carcaça e reduz a espessura de toucinho, sem influenciar a qualidade da carne em suínos.

\section{Agradecimentos}

Ao Conselho Nacional de Desenvolvimento Científico e Tecnológico e à Coordenação de Aperfeiçoamento de Pessoal de Nível Superior, pelas bolsas concedidas.

\section{Referências}

AKAHOSHI,A.; KOBA,K.; ICHINOSE, F.; KANEKO, M.; SHIMODA, A.; NONAKA, K.; IWATA, T.; YAMAUCHI, Y.; TSUTSUMI, K.; SUGANO, M. Dietary protein modulates the effect of CLA on lipid metabolism in rats. Lipids, v.39, p.25-30, 2004.

BASSAGANYA-RIERA， J.; HONTECILLAS-MAGARZO， R.; BREGENDAHL, K.; WANNEMUEHLER, M.J.; ZIMMERMAN, D.R. Effects of dietary conjugated linoleic acid in nursery pigs of dirty and clean environments on growth, empty body composition, and immune competence. Journal of Animal Science, v.79, p.714-721, 2001.

CORINO, C.; MAGNI, S.; PASTORELLI, G.; ROSSI, R.; MOUROT, J. Effect of conjugated linoleic acid on meat quality, lipid metabolism, and sensory characteristics of dry-cured hams from heavy pigs. Journal of Animal Science, v.81, p.2219-2229, 2003.

D'SOUZA, D.N.; MULLAN, B.P. The effect of genotype, sex and management strategy on the eating quality of pork. Meat Science, v.60, p.95-101, 2002. 
DUGAN, M.E.R.; AALHUS, J.L.; KRAMER, J.K.G. Conjugated linoleic acid pork research. American Journal of Clinical Nutrition, v.79, p.1212-1216, 2004.

EGGERT, J.M.; BELURY, M.A.; KEMPA-STECZKO, A.; MILLS, S.E.; SCHINCKEL, A.P. Effects of conjugated linoleic acid on the belly firmness and fatty acid composition of genetically lean pigs. Journal of Animal Science, v.79, p.2866-2872, 2001.

FUNCK, L.G.; BARRERA-ARELLANO, D.; BLOCK, J.M. Ácido linoléico conjugado (CLA) e sua relação com a doença cardiovascular e os fatores de risco associados. Archivos Latinoamericanos de Nutrición, v.56, p.123-134, 2006.

GATLIN, L.A.; SEE, M.T.; LARICK, D.K.; LIN, X; ODLE, J. Conjugated linoleic acid in combination with supplemental dietary fat alters pork fat quality. Journal of Nutrition, v.132, p.3105-3112, 2002.

JOO, S.T.; LEE, J.I.; HA, Y.L.; PARK, G.B. Effects of dietary conjugated linoleic acid on fatty acid composition, lipid oxidation, color, and water-holding capacity of pork loin. Journal of Animal Science, v.80, p.108-112, 2002.

LIN, H.; BOYLSTON, T.D.; CHANG, M.J.; LUEDECKE, L.O.; SHULTZ, T.D. Survey of the conjugated linoleic acid contents of dairy products. Journal of Dairy Science, v.78, p.2358-2365, 1995.

LOVATTO, P.A.; LEHNEN, C.R.; ANDRETTA, I.; CARVALHO, A.D.; HAUSCHILD, L. Meta-análise em pesquisas científicas: enfoque em metodologias. Revista Brasileira de Zootecnia, v.36, p.285-294, 2007.

MINITAB. Minitab: software for quality improvement. Versão 15. Belo Horizonte: Minitab, 2007.

O'QUINN, P.R.; NELSSEN, J.L.; GOODBAND, R.D.; UNRUH, J.A.; WOODWORTH, J.C.; SMITH, J.S.; TOKACH, M.D. Effects of modified tall oil versus a commercial source of conjugated linoleic acid and increasing levels of modified tall oil on growth performance and carcass characteristics of growing-finishing pigs. Journal of Animal Science, v.78, p.2359-2368, 2000.

OSTROWSKA, E.; MURALITHARAN, M.; CROSS, R.F.; BAUMAN, D.E.; DUNSHEA, F.R. Dietary conjugated linoleic acids increase lean tissue and decrease fat deposition in growing pigs. The Journal of Nutrition, v.129, p.2037-2042, 1999.

OSTROWSKA, E.; SUSTER, D.; MURALITHARAN, M.; CROSS, R.F.; LEURY, B.J.; BAUMAN, D.E.; DUNSHEA, F.R. Conjugated linoleic acid decreases fat accretion in pigs: evaluation by dual-energy X-ray absorptiometry. British Journal of Nutrition, v.89, p.219-229, 2003.

PELLEYMOUNTER, M.A.; CULLEN, M.J.; BAKER, M.B.; HECHT, R.; WINTERS, D.; BOONE, T.; COLLINS, F. Effects of the obese gene product on body weight regulation in ob/ob mice. Science, v.269, p.540-543, 1995.
RAMSAY, T.G.; EVOCK-CLOVER, C.M.; STEELE, N.C.; AZAIN, M.J. Dietary conjugated linoleic acid alters fatty acid composition of pig skeletal muscle and fat. Journal of Animal Science, v.79, p.2152-2161, 2001.

SANTOS, L.D. dos; FURUYA, W.M.; MATSUSHITA, M.; SILVA, L.C.R. da; SILVA, T.S. de C.; BOTARO, D. Ácido linoléico conjugado (CLA) em dietas para tilápia-do-nilo: desempenho produtivo, composição química e perfil de ácidos graxos. Revista Brasileira de Zootecnia, v.36, p.1481-1488, 2007.

SANTOS-ZAGO, L.F.; BOTELHO, A.P.; OLIVEIRA, A.C. de. Os efeitos do ácido linoléico conjugado no metabolismo animal: avanço das pesquisas e perspectivas para o futuro. Revista de Nutrição, v.21, p.195-221, 2008.

SAUVANT, D.; SCHMIDELY, P.; DAUDIN, J.J.; ST-PIERRE, N.R. Meta-analyses of experimental data in animal nutrition. Animal, v.2, p.1203-1214, 2008.

THIEL-COOPER, R.L.; PARRISH JUNIOR, F.C.; SPARKS, J.C.; WIEGAND, B.R.; EWAN, R.C. Conjugated linoleic acid changes swine performance and carcass composition. Journal of Animal Science, v.79, p.1821-1828, 2001

WANG, Y.W.; JONES, P.J.H. Dietary conjugated linoleic acid and body composition. American Journal of Clinical Nutrition, v.79, p.1153-1158, 2004.

WEBER, T.E.; RICHERT, B.T.; BELURY, M.A.; GU, Y.; ENRIGHT, K.; SCHINCKEL, A.P. Evaluation of the effects of dietary fat, conjugated linoleic acid, and ractopamine on growth performance, pork quality, and fatty acid profiles in genetically lean gilts. Journal of Animal Science, v.84, p.720-732, 2006.

WEBER, T.E.; SCHINCKEL, A.P.; HOUSEKNECHT, K.L.; RICHERT, B.T. Evaluation of conjugated linoleic acid and dietary antibiotics as growth promotants in weanling pigs. Journal of Animal Science, v.79, p.2542-2549, 2001.

WIEGAND, B.R.; PARRISH, F.C.; SWAN, J.E.; LARSEN, S.T.; BASS, T.J. Conjugated linoleic acid improves feed efficiency, decreases subcutaneous fat, and improves certain aspects of meat quality in stress-genotype pigs. Journal of Animal Science, v.79, p.2187-2195, 2001.

WIEGAND, B.R.; SPARKS, J.C.; PARRISH JUNIOR, F.C.; ZIMMERMAN, D.R. Duration of feeding conjugated linoleic acid influences growth performance, carcass traits, and meat quality of finishing barrows. Journal of Animal Science, v.80, p.637-643, 2002.

ZANINI, S.F.; VICENTE, E.; COLNAGO, G.L.; PESSOTTI, B.M.S.; SILVA, M.A. Manipulation of the fatty acids composition of poultry meat and giblets by dietary inclusion of two oil sources and conjugated linoleic acid. Arquivo Brasileiro de Medicina Veterinária e Zootecnia, v.60, p.1388-1398, 2008.

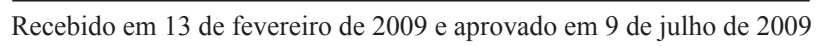

